\title{
Biodynamic optical assay for embryo viability
}

Zhe Li

Natalie Ehmke

Ilka M. Lorenzo

Zoltan Machaty

David Nolte 


\section{Biodynamic optical assay for embryo viability}

\author{
Zhe Li, ${ }^{a, *}$ Natalie Ehmke, ${ }^{b}$ Ilka M. Lorenzo, ${ }^{\mathrm{b}}$ \\ Zoltan Machaty, ${ }^{\mathrm{b}}$ and David Nolte ${ }^{\mathrm{a}}$ \\ aPurdue University, Department of Physics and Astronomy, \\ West Lafayette, Indiana, United States \\ ${ }^{\text {bP }}$ urdue University, Department of Animal Sciences, West Lafayette, \\ Indiana, United States
}

\begin{abstract}
Early stage porcine parthenogenetic embryos were evaluated for metabolic activity using a biodynamic microscope (BDM) that images dynamic light scattering using low-coherence digital holography. The microscope has a 45-deg illumination configuration that reduces specular background for the imaging of small translucent samples. The off-axis illumination is compatible with coherence-gated imaging because of volumetric light scattering in which the coherence plane is tilted at half the illumination angle in a three-dimensional tissue target. The BDM was used to profile the viability of porcine parthenotes with normal and with inhibited mitochondrial adenosine triphosphate (ATP) production using Doppler fluctuation spectroscopy. The ATP concentrations in the parthenotes, which are indicative of developmental potential, were validated by a conventional bioluminescence assay. Biodynamic classifications achieved $\sim 80 \%$ accuracy correlating sample ATP treatment, providing a quick, label-free surrogate measurement to replace invasive metabolic assays as a candidate for evaluating quality of early embryos in the assisted reproductive technology setting. (C) The Authors. Published by SPIE under a Creative Commons Attribution 4.0 Unported License. Distribution or reproduction of this work in whole or in part requires full attribution of the original publication, including its DOI. [DOI: 10.1117/1.JBO.24.6.060502]
\end{abstract}

Keywords: embryo viability; dynamic light scattering; coherent optics; digital holography.

Paper 190140LR received May 1, 2019; accepted for publication May 31, 2019; published online Jun. 25, 2019.

In the United States, 7\% of married women aged 15-44 are infertile, and in-vitro fertilization (IVF) technology is growing at about $5 \%$ per year in terms of IVF cycles. The IVF birth success over all ages of women was only about $30 \%$ in $2016 .{ }^{1,2}$ The IVF success rates are connected to the viability of the blastocyst prior to transfer in utero, and blastocyst quality is related to implantation selection and pregnancy potential. ${ }^{3}$ Because of the low success rates, clinics typically transfer two or three embryos at the same time. However, this can lead to multiple pregnancy and increases the risk of adverse health conditions for both the mother and the offspring. The best practice is to transfer a single high-quality embryo. Morphological grading, using different grading systems, is used to evaluate embryo quality, but it is a subjective process. ${ }^{4}$ Other noninvasive methods include analysis of spent culture media, metabolomics, time-lapse monitoring of embryo development, ${ }^{5}$ and dynamic index variations from

*Address all correspondence to Zhe Li, E-mail: zheli@purdue.edu quantitative phase imaging methods have shown potential to predict embryo viability. ${ }^{6}$ However, there is no reliable evidence from randomized controlled trials to show that these noninvasive techniques are effective in improving the birth rate. ${ }^{7,8}$ Invasive methods may involve biopsy or manipulation of blastocysts 9 and may be detrimental to later development of samples. ${ }^{10} \mathrm{In}$ this letter, we describe the application of biodynamic imaging (BDI) to the problem of viable embryo selection. Biodynamic imaging based on low-coherence Doppler fluctuation spectroscopy, ${ }^{11}$ which captures dynamic activity dominated by intracellular transport, has been used successfully to profile cumulus-oocyte complexes (COCs), ${ }^{12}$ drug response of threedimensional (3-D) cultured tumor spheroids, ${ }^{13,14}$ and to classify cancer patients in preclinical trials. ${ }^{15}$ In this letter, BDI is demonstrated as an accurate surrogate for metabolic assays in embryo selection.

The samples used to perform this study were porcine parthenotes. Parthenotes are embryos derived from oocytes that are activated parthenogenetically by stimuli such as electric shock or exposure to chemicals, which causes them to resume meiosis and stimulates their development. Pig parthenotes undergo cell divisions and can form blastocysts (but are unable to develop to term). Normally, embryos originate from both the male and female gametes that fuse at fertilization, but a parthenote develops from an oocyte only. Nevertheless, parthenotes are frequently used in developmental studies in the laboratory because they behave similarly to IVF embryos. ${ }^{16}$ The parthenote, as a biological model for early embryos, is an ideal model for evaluating a new optical technique for assessing embryo quality.

The biodynamic microscope (BDM) is a stand-alone module, manufactured by Animated Dynamics, Inc., that inserts into a conventional Olympus IX-73 inverted microscope, allowing a user to switch between conventional transillumination microscopy and a BDI mode. The BDM interferometry system is a Mach-Zehnder configuration. A Superlum ${ }^{\mathrm{TM}}$ superluminescent diode centered at $841.2 \mathrm{~nm}$ with a $28.9-\mathrm{nm}$ bandwidth and 20.9-mW output power was used as the light source that was incident on the sample at a 45-deg angle relative to the optic axis of the collection objective lens. The translation stage controls the coherence gate based on optical path length (OPL). In conventional backscatter coherence-gated holography, the optical section plane is perpendicular to the optic axis. However, the 45-deg illumination, combined with the three-dimensional volumetric scattering, creates a coherence plane that is tilted at $22.5 \mathrm{deg}$ (half of the illumination angle), and the sample "flythrough" occurs at this oblique angle when translating the reference mirrors. The advantages of this BDM design are the reduction of specular background reflections and the elimination of the conventional beamsplitter normally used in the backscatter configuration to improve optical brightness of dim translucent samples. The oblique coherence plane needs minor additional digital postprocessing after holographic reconstruction to regain a balanced aspect ratio of the volumetric target. The optical configuration is shown in Figs. 1(a) and 1(b). The optical design of the BDM uses an Olympus UPLFLN $4 \times$ objective lens with a 0.13-numerical aperture, 17-mm working distance, and 45-mm parfocal distance. The BDI mode has a 0.05-numerical aperture, up to 1-mm field of view (determined by the illumination beam size), and a pixel size of $8 \mu \mathrm{m}$. The $\mathrm{BDM}$ is placed on a vibration isolation platform to minimize the mechanical disturbance. 

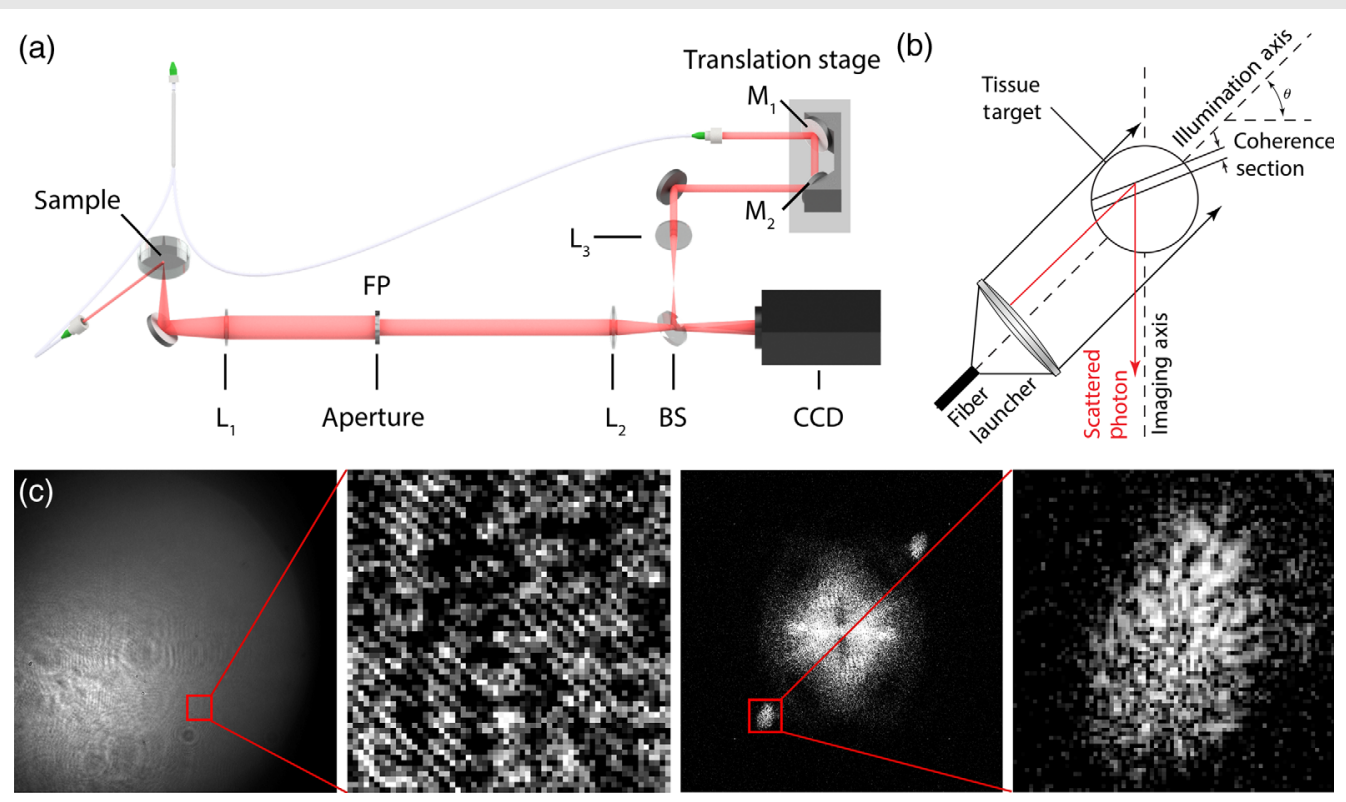

Fig. 1 (a) System setup of the BDI mode of the BDM. $L_{1}$ is the Fourier transform lens, $L_{2}$ is the imaging lens, $L_{3}$ is the phase compensation lens, and the CCD is on the Fourier domain. The delay stage with mirrors $M_{1}$ and $M_{2}$ is used for adjusting the OPL of the reference arm. FP, Fourier plane; BS, beamsplitter. (b) Illustration of the 45-deg illumination angle and 22.5-deg coherence-gated section. (c) Sample hologram reconstruction, from left to right: raw hologram, enlarged fringes (background subtracted for better contrast), two-dimensional FFT, and image with dynamic speckles.

Ovaries were collected from slaughtered pigs, and follicular contents were aspirated from individual follicles in the lab using a hypodermic needle attached to a $10-\mathrm{mL}$ syringe. The oocytes with attached cumulus cells (COCs) were collected from the follicular fluid by means of a stereomicroscope, washed in TL-HEPES medium, and good-quality oocytes were selected for in-vitro maturation in the appropriate medium. Matured COCs were placed in $0.1 \%$ hyaluronidase, vortexed until denuded and washed in TL-HEPES medium. Denuded oocytes were then placed in electroporation medium in a chamber containing two stainless steel electrodes 0.5 -mm apart. Parthenogenetic development was induced via electroporation with two direct-current pulses 1-s apart of $1.2 \mathrm{kV} / \mathrm{cm}, 60 \mu$ s each via a CF-150/B cell fusion instrument. After electric stimulation, prospective embryos were rinsed in TL-HEPES medium and placed in $20-\mu \mathrm{L}$ droplets of PZM-3 medium with or without $20-\mu \mathrm{M}$ sodium azide $\left(\mathrm{NaN}_{3}\right)$. When an embryo is cultured with dilute sodium azide $\left(\mathrm{NaN}_{3}\right)$ at the 1-cell stage, the azide ion $\left(\mathrm{N}_{3}^{-}\right)$ inhibits the electron transport in the mitochondrial membrane and prevents oxidative phosphorylation. Mitochondrial adenosine triphosphate (ATP) production of the embryo is thus inhibited, and its ability to develop into a healthy blastocyst is significantly reduced. ${ }^{17,18}$ Each droplet contained 10 embryos, the droplets were covered with light mineral oil to prevent evaporation, and culture dishes containing the droplets were placed in a $\mathrm{CO}_{2}$ incubator. Both groups were allowed to culture in their respective medium up to $96 \mathrm{~h}$, at $39^{\circ} \mathrm{C}$, under $5 \% \mathrm{CO}_{2}$ in air.

After the culture period, the embryos that had reached the early morula stage were selected for assessment. For BDI measurements, samples were prepared with two protocols with different immobilization methods: (1) each sample was prepared in a 50- $\mu$ L TL-HEPES droplet on a plate treated with Cell-Tak ${ }^{\mathrm{TM}}$ and covered with mineral oil, or (2) three washing dishes of TLHEPES medium [without polyvinyl alcohol (PVA)] were prepared, and each sample was washed in each of the dishes and plated in 50- $\mu$ L PVA-free TL-HEPES droplet and covered with mineral oil. The plate was heated with the temperature maintained at a physiological $39^{\circ} \mathrm{C}$. A complete BDI dataset for a sample contained 100 background frames and 2500 holograms, captured at $25 \mathrm{fps}$ with an exposure time of $20 \mathrm{~ms}$, reconstructed with Fourier transform [Fig. 1(c)]. The coherence gate was placed approximately at the middle of the sample at a depth of about $100 \mu \mathrm{m}$ from the embryo surface. The BDI measurement finished in a short time ( 1 to 2 min of sample preparation and $2 \mathrm{~min}$ of data acquisition), by placing the samples within a safe light exposure range and a non- $\mathrm{CO}_{2}$-controlled environment. After the BDI measurement, each embryo was lysed in $10 \mu \mathrm{L}$ of RIPA lysis and extraction buffer and stored for 10 min on ice. ATP measurements were then performed using Molecular Probes ${ }^{\mathrm{TM}}$ ATP Determination Kit on a Spark ${ }^{\mathrm{TM}}$ 10M multimode microplate reader. A standard curve was obtained based on bioluminescence from the reactions of standard solutions with known ATP amount. Bioluminescence of each sample was measured and compared against the standard curve and converted to the respective ATP amount of substance. A total of 133 embryo samples measured on 12 separate days were used in the analysis, consisting of 85 control samples and $48 \mathrm{NaN}_{3}$ treated samples. The BDI data for the samples with different immobilization methods were normalized and combined in the analysis.

Samples are assessed using the BDM by evaluating speckle fluctuation properties. The average backscatter brightness (BB, i.e., intensity) and normalized standard deviation (NSD) for each pixel are calculated over 500 frames (NSD is defined as $\Delta I /\langle I\rangle$, where $\Delta I$ is the standard deviation of intensity and $\langle I\rangle$ is average intensity). Each sample is characterized by optical coherence imaging ${ }^{19}$ (OCI) shown in Fig. 2(b), by motility contrast imaging ${ }^{20}$ (MCI) shown in Fig. 2(c), and by the Doppler power spectrum ${ }^{21}$ shown in Figs. 2(d) and 2(e). Sample spectra 

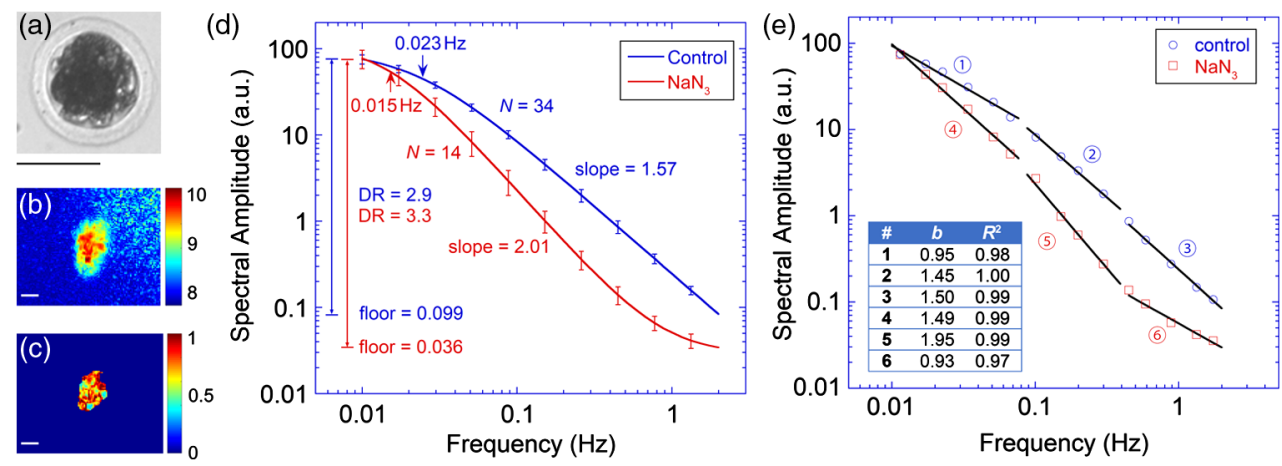

Fig. 2 (a) Microscope image of a parthenote sample captured in transillumination. (b) OCl of the sample. Color map is on log scale. (c) $\mathrm{MCl}$ of the same sample. The value of each pixel is NSD (scale bar $=100 \mu \mathrm{m}$ in a, b, and c). (d) Averaged fluctuation spectra (smoothed) of control samples and $\mathrm{NaN}_{3}$-treated samples plated in PVA-free TL-HEPES droplets, with different knee frequencies, slopes, Nyquist floors, and dynamic ranges ("DR") on the log-log scale. Standard errors are used as values of error bars. (e) An example of three-segment linear fitting for spectra. (Markers are a subset of data points with even intervals.)

span a range of 0.01 to $2 \mathrm{~Hz}$ and are fit with a "stretched" Lorentzian lineshape

$S=\frac{A}{\omega_{0}^{s}+\omega^{s}}+N_{y}$,

where $\omega_{0}$ is the knee frequency (the "roll-over" frequency of the spectrum, i.e., where the curvature of the curve changes), $s$ is the slope in the midfrequency range, and $N_{y}$ is the Nyquist floor. These parameters are the potential biomarkers of embryo viability. Typically, an azide-treated spectrum displays a lower knee frequency, which is correlated with slower intracellular activities. Azide-treated spectra also have a greater dynamic range (DR) and a steeper midfrequency slope on a log-log power spectrum graph. In addition, the spectrum is characterized by the slope and $R^{2}$ values from linear fittings on the log scale (i.e., a power law fitting model $S=a x^{-b}$ ), both "globally" on the entire spectrum and "locally" in three frequency ranges: 0.01 to $0.08 \mathrm{~Hz}, 0.08$ to $0.4 \mathrm{~Hz}$, and 0.4 to $2 \mathrm{~Hz}$. Values of $b$ and $R^{2}$ are used to describe the spectrum shape (values for the three ranges use the notations $b_{1}, b_{2}, b_{3}, r_{1}, r_{2}$, and $r_{3}$ ). Typical values for these parameters across a range of parthenotes are listed in Table 1.

Analysis of the sample ATP content shows that the controlgroup parthenotes have significantly more ATP than $\mathrm{NaN}_{3}$ treated samples [shown in Fig. 3(a) with a $p$-value of $p<10^{-3}$ ], which signifies that the control group of parthenotes are metabolically more active. Therefore, a test was run to determine if sample treatment is predictive of sample ATP content with the control group labeled as condition positive, and ATP content was used as the score. ATP content greater than a threshold value was predicted as positive and ATP content less than that value was predicted as negative. A true-positive rate (TPR) and a false-positive rate (FPR) were calculated for each threshold, and an receiver operating characteristic curve with the threshold as the varying parameter as shown in Fig. 3(b). This classifier achieved $89.7 \%$ accuracy at an ATP threshold of $5 \mathrm{nmol}$.

In this correlative study, biomarkers were used to build sample classifications and to estimate the predictive ability of the BDM assay to select embryos with high metabolic activity. A feature vector contains the key biodynamic biomarkers that represent sample properties with 13 elements (BB, NSD, knee, midfrequency slope, $R^{2}$ value of spectrum, floor, $\mathrm{DR}$, and $b$ and $R^{2}$ values from three frequency ranges). Principal component analysis (PCA) was used for dimensionality reduction, after which a quadratic-kernel support vector machine (SVM) was used to predict the sample group (using MATLAB ${ }^{\circledR}$ Statistics and Machine Learning Toolbox ${ }^{\mathrm{TM}}$ ). In this analysis, the "control" group was defined as condition positive, while the $\mathrm{NaN}_{3}$-treated samples were defined as condition negative. A fivefold cross validation was used to characterize the performance of the classifier, where a classification score, indicating a signed distance from the observation to the decision boundary, was assigned to each sample. Choosing a fivefold cross validation ensured that the test groups were large enough (with $n \sim 26$ in each group) and that the bias was low. The fivefold cross validation was run 10 times, and in each run TPR and FPR values were calculated with varying score thresholds, creating the receiver operating characteristic (ROC) curves plotted in Fig. 3(c). The SVM classifier performed with an area under the curve (AUC) of 0.812 and an accuracy of $79.3 \%$.

In conclusion, BDI of intracellular activity has been demonstrated on early stage parthenotes as biologically relevant models of natural embryos. The biodynamic assay performs as a surrogate for invasive ATP assays and can distinguish parthenotes that have high metabolic activity from parthenotes that have compromised metabolism. This assay is noninvasive and can be performed longitudinally to track embryo health while preserving embryo viability. This optical technique has the

Table 1 Typical values for biodynamic biomarkers.

\begin{tabular}{lccccccccccc} 
Quantity & $A$ & $\omega_{0}(\mathrm{~Hz})$ & $s$ & $N_{y}$ & $r$ & $b_{1}$ & $r_{1}$ & $b_{2}$ & $r_{2}$ & $b_{3}$ & $r_{3}$ \\
\hline Value & 0.04 & 0.012 & 1.86 & 1.01 & 0.97 & 0.97 & 0.93 & 1.54 & 0.98 & 1.09 & 0.97 \\
\hline
\end{tabular}




\section{JBO Letters}
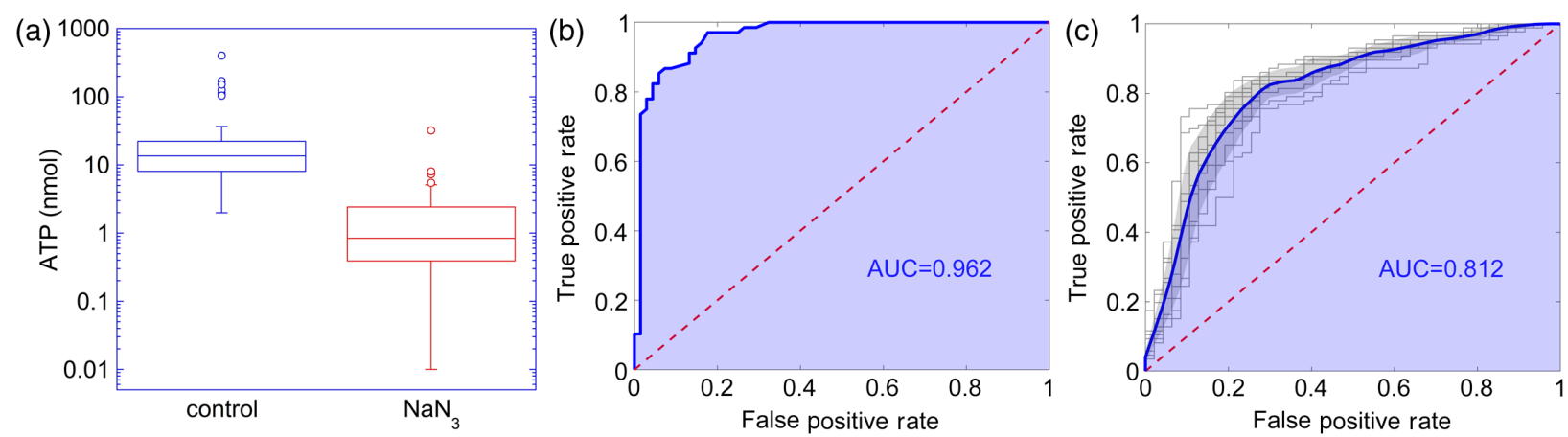

Fig. 3 (a) Box plot of ATP content in "control" parthenotes and $\mathrm{NaN}_{3}$-treated parthenotes. (b) ROC curve of the sample ATP biochemical assay and (c) ROC curves and averages from 10 runs of the fivefold cross-validated SVM classifier on the biodynamic feature vector. The blue curve is the average of TPRs for each FPR, while the gray area is the \pm 1 standard deviation of the TPRs.

potential to improve IVF success rates. Future studies will include a prospective preclinical trial during which porcine embryos will be evaluated with BDI and then transferred in utero in sows to establish pregnancy rates for BDI-selected transfer relative to conventional selection.

\section{Disclosures}

Professor Nolte has a financial interest in Animated Dynamics, Inc., that provided the biodynamic microscope used for this project, and Professor Machaty is a paid consultant.

\section{Acknowledgments}

This letter was supported by grant from the National Institutes of Health (NIH) (R01-HD078682).

\section{References}

1. Centers for Disease Control and Prevention, American Society for Reproductive Medicine, Society for Assisted Reproductive Technology, 2016 Assisted Reproductive Technology Fertility Clinic Success Rates Report, Atlanta (GA): US Dept of Health and Human Services (2018).

2. Centers for Disease Control and Prevention, American Society for Reproductive Medicine, Society for Assisted Reproductive Technology, 2016 Assisted Reproductive Technology National Summary Report, Atlanta (GA): US Dept of Health and Human Services (2018).

3. B. Balaban et al., "Blastocyst quality affects the success of blastocyststage embryo transfer," Fertil. Steril. 74(2), 282-287 (2000).

4. P. W. Farin et al., "Agreement among evaluators of bovine embryos produced in vivo or in vitro," Theriogenology 44(3), 339-349 (1995).

5. P. Kovacs, "Embryo selection: the role of time-lapse monitoring," Reprod. Biol. Endocrinol. 12(1), 124 (2014).

6. T. H. Nguyen et al., "Gradient light interference microscopy for 3D imaging of unlabeled specimens," Nat. Commun. 8(1), 210 (2017).

7. C. Racowsky, P. Kovacs, and W. P. Martins, "A critical appraisal of timelapse imaging for embryo selection: where are we and where do we need to go?" J. Assisted Reprod. Genet. 32(7), 1025-1030 (2015).
8. Y. G. Wu et al., "Different effectiveness of closed embryo culture system with time-lapse imaging (EmbryoScope ${ }^{\mathrm{TM}}$ ) in comparison to standard manual embryology in good and poor prognosis patients: a prospectively randomized pilot study," Reprod. Biol. Endocrinol. 14(1), 49 (2016).

9. G. Kakourou et al., "Investigation of gene expression profiles before and after embryonic genome activation and assessment of functional pathways at the human metaphase II oocyte and blastocyst stage," Fertil. Steril. 99(3), 803-814.e23 (2013).

10. K. Kirkegaard, J. J. Hindkjaer, and H. J. Ingerslev, "Human embryonic development after blastomere removal: a time-lapse analysis," Hum. Reprod. 27(1), 97-105 (2012).

11. Z. Li et al., "Doppler fluctuation spectroscopy of intracellular dynamics in living tissue," J. Opt. Soc. Am. A 36(4), 665-677 (2019).

12. R. An et al., "Biodynamic imaging of live porcine oocytes, zygotes and blastocysts for viability assessment in assisted reproductive technologies," Biomed. Opt. Express 6(3), 963-976 (2015).

13. H. Sun et al., "Biodynamic imaging for phenotypic profiling of threedimensional tissue culture," J. Biomed. Opt. 22(1), 016007 (2017).

14. D. D. Nolte et al., "Tissue dynamics spectroscopy for three-dimensional tissue-based drug screening," J. Lab. Autom. 16(6), 431-442 (2011).

15. H. Choi et al., "Biodynamic digital holography of chemoresistance in a pre-clinical trial of canine B-cell lymphoma," Biomed. Opt. Express 9(5), 2214-2228 (2018).

16. A. Paffoni et al., "Parthenogenetic activation: biology and applications in the ART laboratory," Placenta 29, 121-125 (2008).

17. J. Thompson et al., "Effect of inhibitors and uncouplers of oxidative phosphorylation during compaction and blastulation of bovine embryos cultured in vitro," J. Reprod. Fertil. 118(1), 47-55 (2000).

18. Z. Macháty et al., "Inhibitors of mitochondrial ATP production at the time of compaction improve development of in vitro produced porcine embryos," Mol. Reprod. Dev 58(1), 39-44 (2001).

19. D. D. Nolte, Optical Interferometry for Biology and Medicine, pp. 312-216, Springer, New York (2012).

20. K. Jeong, J. J. Turek, and D. D. Nolte, "Imaging motility contrast in digital holography of tissue response to cytoskeletal anti-cancer drugs," Opt. Express 15, 14057-14064 (2007).

21. K. Jeong, J. J. Turek, and D. D. Nolte, "Speckle fluctuation spectroscopy of intracellular motion in living tissue using coherence-domain digital holography," J. Biomed. Opt. 15(3), 030514 (2010). 